\title{
Analyzing block type channel estimation for OFDM based digital communication system
}

\author{
Mandeep Kaur ${ }^{1, a}$ and Savina Bansal ${ }^{1}$ \\ Deptt of ECE, GZSCCET, Maharaja Ranjit Singh State Technical University, Bathinda
}

\begin{abstract}
Orthogonal frequency division multiplexing (OFDM) is a promising technique in the current broadband wireless communication system due to the high data rate transmission capability and the ability to combat frequency selective fading of the channel. Channel estimation is mainly implemented by sending pilot symbols in the transmitted bit streams. In this paper, channel estimation based on block type pilot arrangements is analyzed using Least Square (LS) and Minimum Mean Square Error (MMSE) channel estimators. Performance is analyzed in terms of Bit Error Rate and Mean Square Error by varying pilot energy levels and by varying channel length. It is gathered that performance gets affected considerably with change in pilot energy levels implying there exist an optimum value for pilot energy for getting better performance.
\end{abstract}

\section{Introduction}

In OFDM system many sub-channels are used in parallel. The channels are overlapping in frequency, but the distance between them is chosen so that the different channels anyhow are orthogonal. OFDM is a promising technique in the current broadband wireless communication system due to the high data rate transmission capability and the ability to combat frequency selective fading of the channel. OFDM, which is the recent trend in wireless technology, is a multicarrier modulation scheme having high data stream splitting into low data stream that are transmitted simultaneously over a number of subcarriers. OFDM is widely used in the wireless systems such as wireless LAN, terrestrial digital television broadcasting, cell-phone and Wi-MAX.

Wireless channels used for transmitting the high data rate digital signals usually suffers from various impairments due to multipath propagation of the signals owing to different types of obstacles present, and frequency dependent channel characteristics. As a result the received signal gets corrupted leading to misjudgment of the signal transmitted and hence reducing the system fidelity and utility for high data rate transmissions. To recover the signal correctly at the receiving end it becomes crucial to apply the inverse channel characteristics at the receiver to nullify the non-linear channel effects. So, channel estimation is an important aspect of high speed data transmission systems before applying demodulation at the receiving end. Channel estimation is mainly implemented by sending pilot symbols in the transmitted bit streams. The channel estimation has been performed by inserting pilot tones into each OFDM symbol. In this paper, channel estimation based on block type pilot arrangements using LS and MMSE channel estimators is studied. This paper is organized as follows. In this section 2, simulated system description is described. Section 3 discusses channel estimation. Performance analysis and conclusion are given in section 4 and section 5 .

\section{Simulated system description}

In figure 1, the information data in binary form are first grouped and mapped into mutiamplitude multi-phase signals according to the type of modulation used in the signal modulator. After inserting pilots uniformly between the information data sequence, IFFT block is used to transform and multiplex the complex data sequence into time domain signal. Following the IFFT block, a guard interval (larger than the expected delay spread), is inserted in order to prevent possible intersymbol interference (ISI) in OFDM systems. The transmitted signal is then sent to a frequency selective multi-path time varying slow fading channel.

At the receiver, the guard insertion is removed first and the received samples are then sent to the FFT block for de-multiplexing the multi-carrier signal. Following FFT block, the pilot signals are extracted from the demultiplexed samples. The transmitted data samples can then be recovered from the knowledge of the channel responses by simply dividing the received signal by the channel response. After signal demodulation at the demodulator, the binary data could be reconstructed at the receiver output.

\footnotetext{
a Corresponding author: mandeepdhanoa23@gmail.com
} 

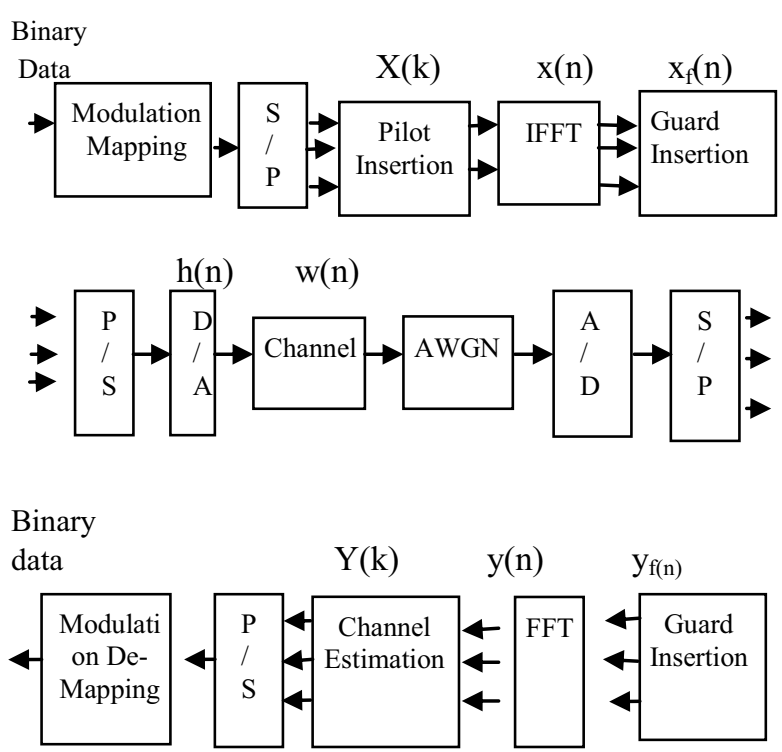

Figure 1. Simulated OFDM system

\section{Channel estimation}

In block type pilot arrangement, OFDM symbols with pilots at all subcarriers are transmitted periodically for channel estimation. Block type pilot arrangement is especially suitable for slow fading radio channels. This type of pilot arrangement is relatively insensitive to the frequency selectivity.

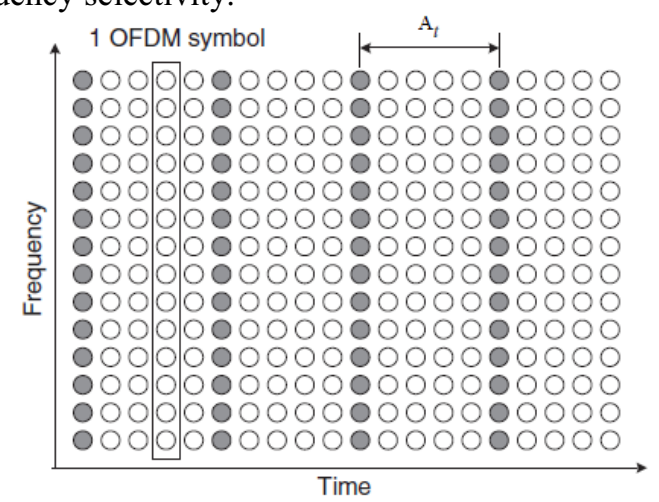

Figure 2. Block type pilot arrangement

\subsection{Channel estimation based on block type arrangement}

To estimate the channel conditions (specified by $\mathrm{H}$ ) given the pilot signals (specified by $\mathrm{X}$ ) and received signals (specified by Y), with or without using certain knowledge of the channel statistics. The receiver uses the estimated channel conditions to decode the received data inside the block until the next pilot symbol arrives. The estimation can be based on least square (LS) and minimum mean square error (MMSE) estimators.

\section{$>\mathbf{L S}$}

The LS estimator minimizes the weighted errors between the measurements and model. The LS estimates $\mathrm{H}$, given the received data $\mathrm{Y}$ and the transmitted symbols $\mathrm{X}$ is given by:

$$
H_{L S}=X^{-1} Y \quad k=0,1, \ldots \ldots, N-1
$$

The main advantage of LS estimator is that it has low complexity but it has high MSE.

\section{$>$ MMSE}

The MMSE estimator employs the second order statistics of the channel conditions to minimize the MSE. Denote by $R_{h h}, R_{H H} \& R_{Y Y}$ the auto covariance matrix of h, $\mathrm{H}$ and $\mathrm{Y}$ respectively and by $R_{h Y}$ the cross covariance matrix between $\mathrm{h}$ and Y. Also denote by $\sigma^{2}$ the noise variance. Assume the channel vector and the noise are uncorrelated, it is derived that:

$$
\begin{aligned}
& R_{H H}=E\left\{H H^{H}\right\}=F R_{h h} F^{H} \\
& R_{h Y}=E\left\{h Y^{H}\right\}=R_{h h} F^{H} X^{H} \\
& R_{Y Y}=X F R_{h h} F^{H} X^{H}+\sigma^{2} I
\end{aligned}
$$

The FFT matrix F is given by:

$$
\left[\begin{array}{ccc}
W_{N}^{00} & \cdots & W_{N}^{0(N-1)} \\
\vdots & \ddots & \vdots \\
W_{N}^{(N-1) 0} & \cdots & W_{N}^{(N-1)(N-1)}
\end{array}\right]
$$

Where

$$
W_{N}^{n k}=\frac{1}{N} e^{-j 2 \pi\left(\frac{n}{N}\right) k}
$$

Assume $R_{h h}$ (thus $R_{H H}$ ) and $\sigma^{2}$ are known at the receiver in advance, the MMSE estimator of $h$ is given by:

$h_{M M S E}=R_{h Y} R_{Y Y}^{-1} Y_{H H}$

Note that if $\mathrm{h}$ is not Gaussian, $h_{M M S E}$ is not necessary a MMSE estimator.

$$
\begin{aligned}
H_{M M S E} & =F h_{M M S E} F\left[\left(F^{H} X^{H}\right)^{-1} R_{h h}^{-1} \sigma^{2}+X F\right]^{-1} Y \\
& =F R_{h h}\left[\left(F^{H} X^{H} X F\right)^{-1} \sigma^{2}+R_{h h}\right] F^{-1} H_{L S} \\
& =R_{H H}\left[R_{h H}+\sigma^{2}\left(X X^{H}\right)^{-1}\right]^{-1} H_{L S}
\end{aligned}
$$

MMSE tries to minimize the MSE error rate. It has better rate than LS estimator having high SNR ratio.

\section{Performance analysis}

Channel estimation based on block based pilot arrangement using LS and MMSE channel estimators has been implemented for OFDM system using MATLAB platform.

Table 1. Performance parameters analysis

\begin{tabular}{|l|l|}
\hline $\begin{array}{l}\text { Number of sub } \\
\text { channels }\end{array}$ & 256 \\
\hline
\end{tabular}




\begin{tabular}{|l|l|}
\hline $\begin{array}{l}\text { Number of } \\
\text { pilots }\end{array}$ & $256 / 8$ \\
\hline Guard interval & $\begin{array}{l}\text { Number of sub } \\
\text { channels/4 }\end{array}$ \\
\hline $\begin{array}{l}\text { Modulation } \\
\text { index, M }\end{array}$ & 4 \\
\hline $\begin{array}{l}\text { Channel } \\
\text { Length }\end{array}$ & 16,32 \\
\hline $\begin{array}{l}\text { Pilot energy } \\
\text { levels }\end{array}$ & $0.5,2,4,6$ \\
\hline
\end{tabular}

The figure 3 shows the BER for BPSK modulation in which the channel estimation is done using LS algorithm at different pilot energy levels. It is seen that performance gets affected considerably with change in pilot energy levels. For very low energy level $(=0.5)$ the BER deteriorates. It improves with increasing pilot energy $(=2)$. However, further increase in energy results in increasing the bit error rate. This implies that there exist an optimum value for pilot energy for getting better performance.

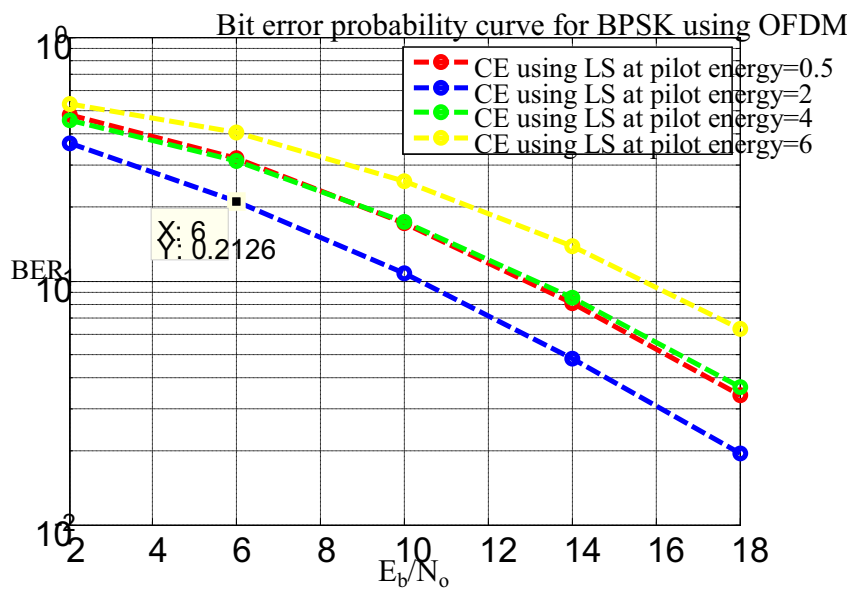

Figure 3. Performance at different pilot energy levels using LS algorithm, $\mathrm{L}=16$

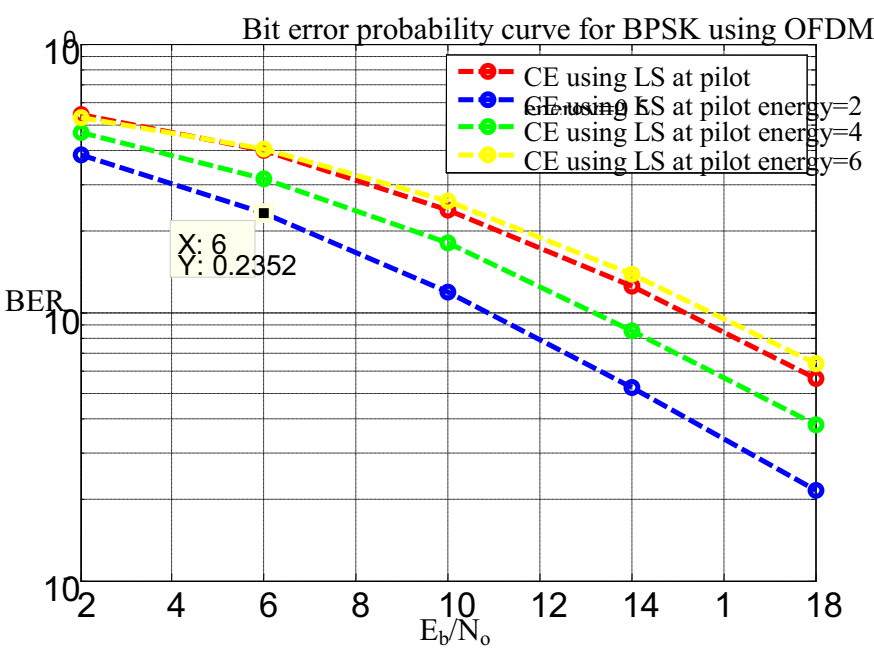

Figure 4. Performance at different pilot energy levels using LS algorithm, $\mathrm{L}=32$

The figure 4 shows the BER rate for BPSK modulation in which the channel estimation is done using LS algorithm at different pilot energy levels and at channel length $\mathrm{L}=32$. It is seen that performance gets affected considerably with change in pilot energy levels. For very low energy level $(=0.5)$ the BER deteriorates. With increase in pilot energy level beyond $\mathrm{E}=0.5$, the performance tends to improve with increase in energy level upto $E=2$. However, further increase in energy level does with help rather BER starts deteriorates as shown for pilot energy $E=4,6$. Though the BER remains slighty better then that at $\mathrm{E}=0.5$. So, $\mathrm{E}=2$ pilot energy level seem to be the optimum one.

Futher, it is seen that with increase in channel length, for given energy per bit to noise power spectral density ratio, BER is slight higher as compared to lower channel length.

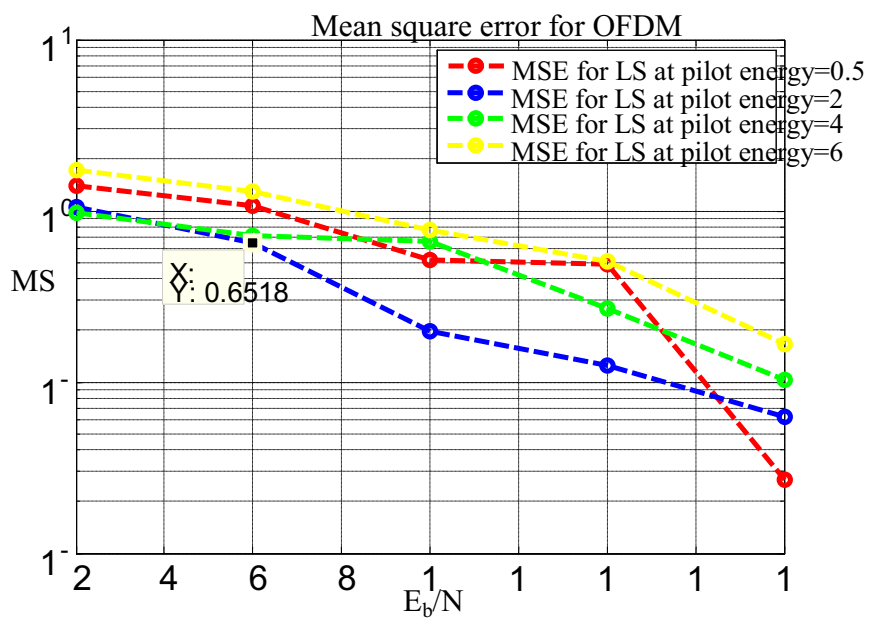

Figure 5. MSE at different pilot energy levels using LS algorithm, $\mathrm{L}=16$

The figure 5 shows the MSE for OFDM using LS algorithm at different pilot energy levels $(=0.5,2,4,6)$. It is seen that MSE for low pilot energy level $(=0.5)$ is higher than that at energy level $(=2)$. However, beyond pilot energy level $(=2)$, the MSE starts increasing. The MSE at pilot energy level (=6), it is highest among all.

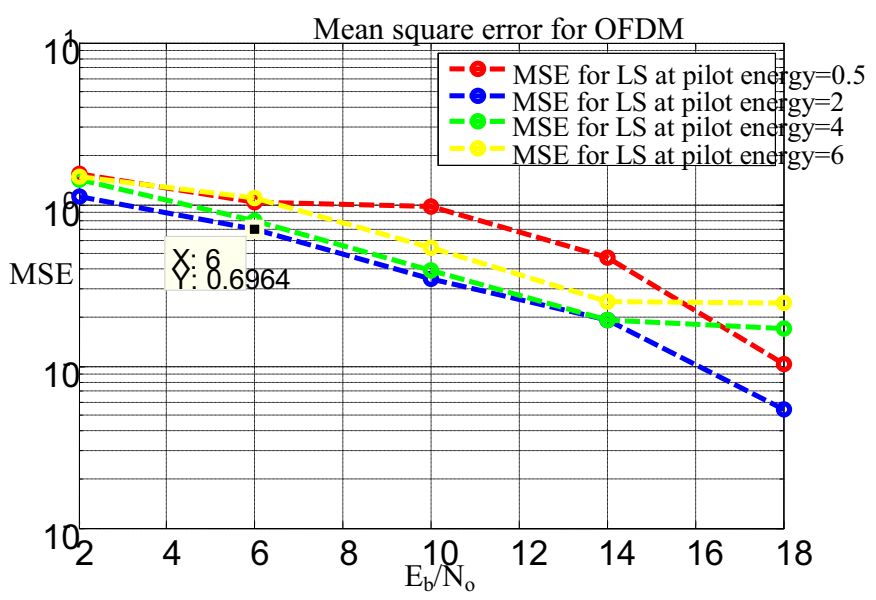

Figure 6. MSE at different pilot energy levels using LS algorithm, $\mathrm{L}=32$

The figure 6 shows the MSE for OFDM using LS algorithm at different energy levels. It is seen that performance for MSE gets affected considerably with change in pilot energy levels. For very low energy level $(=0.5)$ the MSE deteriorates. It improves with 
increasing pilot energy $(=2)$. The MSE for low pilot energy level $(=0.5)$ is higher then that at energy level $(=2)$. However, beyond pilot energy level $(=2)$, the MSE starts increasing. The MSE at pilot energy level $(=0.5)$, it is highest among all.

With increasing $\mathrm{E}_{\mathrm{b}} / \mathrm{N}_{\mathrm{o}}$ ratio, the MSE tends to decrease which indicates there exists a trade-off between MSE and $\mathrm{E}_{\mathrm{b}} / \mathrm{N}_{\mathrm{o}}$.

The figure 7 shows the BER vs $E_{b} / N_{o}$ for BPSK modulation in which the channel estimation is done using MMSE algorithm at pilot energy level $(=2)$. It is seen that BER tend to reduce with increase $E_{b} / N_{0}$. At all pilot energy levels the BER for channel estimation using MMSE is considerably same. The result shows here are for channel length $(\mathrm{L}=16)$ at pilot energy level $(=2)$.

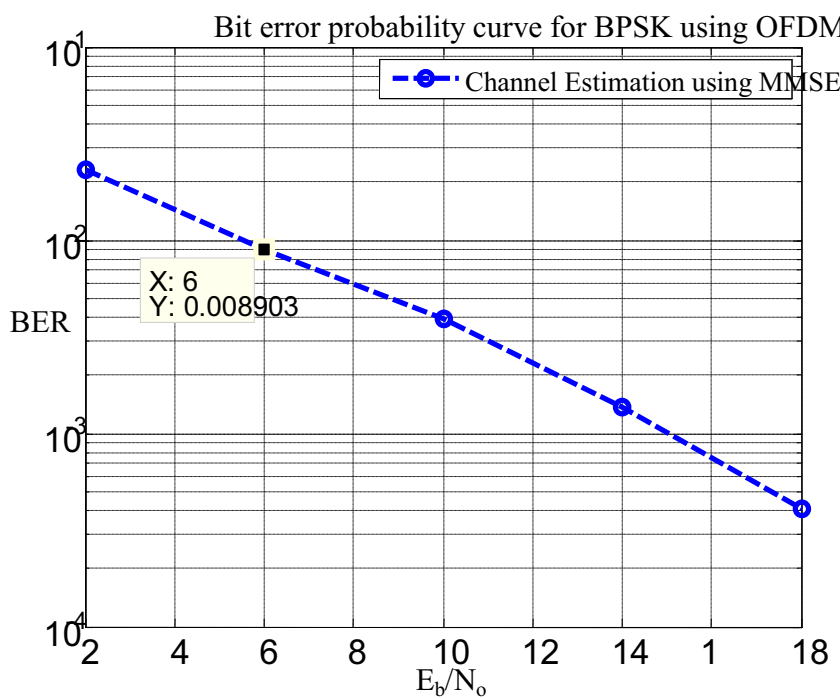

Figure 7. BER at pilot energy level 2 using MMSE algorithm, $\mathrm{L}=16$

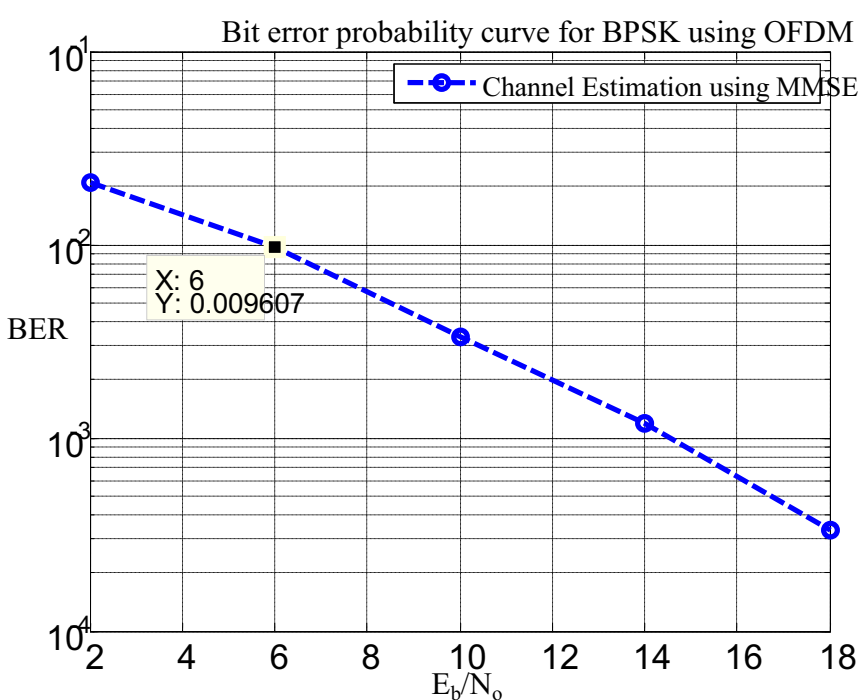

Figure 8. BER at pilot energy level 2 using MMSE algorithm, $\mathrm{L}=32$

The figure 8 shows the BER vs $\mathrm{E}_{\mathrm{b}} / \mathrm{N}_{\mathrm{o}}$ for $\mathrm{BPSK}$ modulation in which the channel estimation is done using MMSE algorithm at pilot energy level $(=2)$. It is seen that BER tend to reduce with increase $E_{b} / N_{0}$. The results shows for channel length $(\mathrm{L}=32)$ at pilot energy level (=2) using MMSE algorithm.

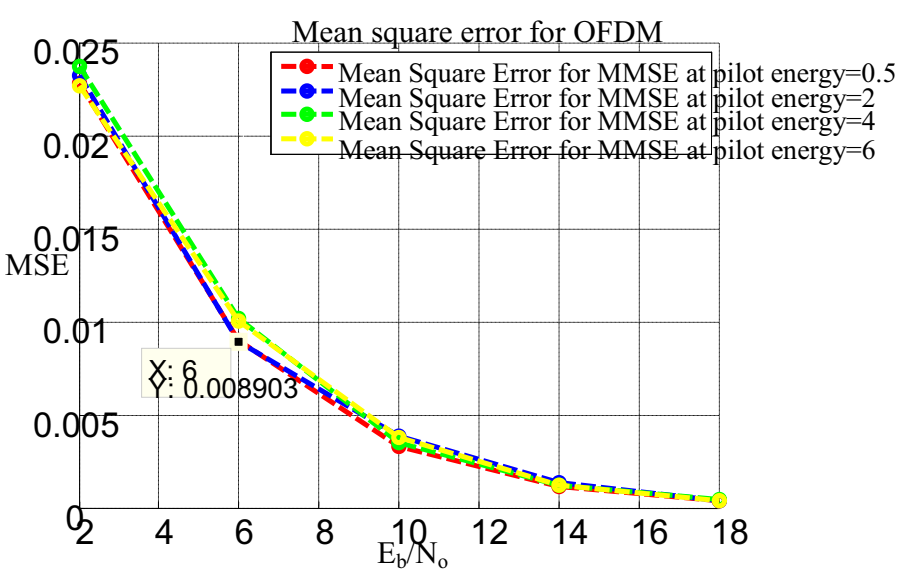

Figure 9. MSE at different pilot energy levels using MMSE algorithm, $\mathrm{L}=16$

The figure 9 shows the MSE for OFDM using MMSE estimator at different pilot energy levels. It is seen that the MSE considerably increases by increasing pilot energy levels $(0.5,2,4,6)$ but MSE tends to reduce with increase in $E_{b} / N_{o}=4,6,8$. With further increase in $E_{b} / N_{0}$, the MSE at pilot energy levels $(0.5,2,4,6)$ is almost same.

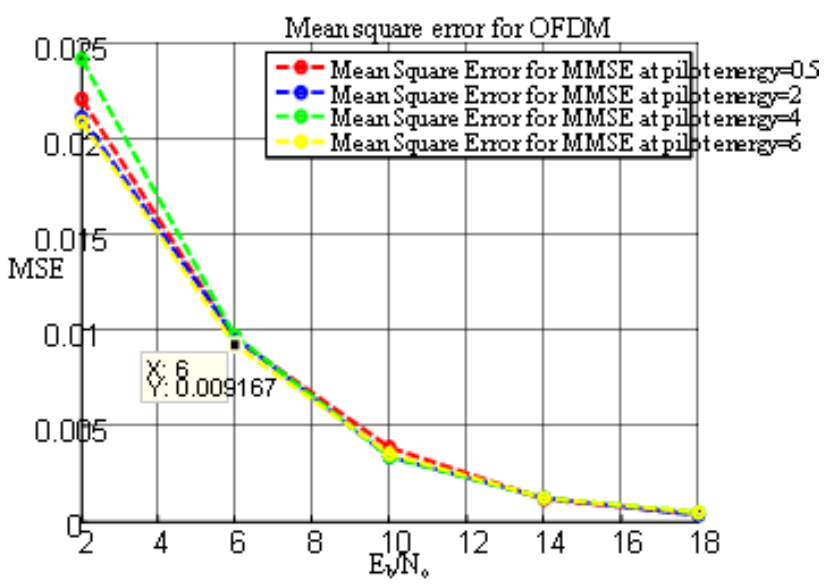

Figure 10. MSE at different pilot energy levels using MMSE algorithm, $\mathrm{L}=32$

The figure 10 shows the MSE for OFDM using MMSE estimator at different pilot energy levels. It is seen that at pilot energy levels $(0.5,2,4,6)$ the MSE tends to reduce with increase in $\mathrm{E}_{\mathrm{b}} / \mathrm{N}_{\mathrm{o}}$. Further increase in $\mathrm{Eb} / \mathrm{No}(=12$, 14,16), the MSE is almost same at all energy levels.

In comparison to similar results for LS algorithm, it is seen that BER is much lower in MMSE algorithm. Although, this improvement is at the cost of increase in complexity.

\section{Conclusion}

In this paper, channel estimation based on block type pilot arrangements is studied and analyzed using Least Square (LS) and Minimum Mean Square Error (MMSE) channel estimators. The channel estimation has been performed by inserting pilot tones into each OFDM symbol. Based on the results obtained, it is gathered that 
performance gets affected considerably with change in pilot energy levels. For very low energy level $(=0.5)$ the BER and MSE deteriorates. It improves with increasing pilot energy $(=2)$, though further increase is not helpful and performance degrades. With increasing channel length, the BER and MSE tends to increase slightly.

\section{References}

1. Stuti Ramola, "Digital Communication-Technology and Advancements", Advance in Electronic and Electric Engineering. ISSN 2231-1297, Volume 4, Number 4 (2014), pp. 367-374.

2. Anshul C.Gurve, S.S.Wasnik, A.D.Bijwe, Ashwini S. Yerlekar, Nilesh Chide, "Study of OFDM Variants and Implementation of OFDM Using fft / ifft", International Journal of Advanced Research in Computer and Communication Engineering Vol. 3, Issue 7, July 2014.

3. Srishtansh Pathak and Himanshu Sharma, "Channel Estimation in OFDM Systems", International Journal of Advanced Research in Computer Science and Software Engineering, Volume 3, Issue 3, March 2013, pp.312-327.

4. Lokesh Panchal, Manjil Upadhyay, Nirali Shah, Aniruddh Amin, "Significant Implementation of LS and MMSE Channel Estimation for OFDM Technique", Int. Journal of Engineering Research and Applications, Vol. 4, Issue 3 (Version 6), March 2014, pp.38-41.

5. Chaudhari Sachin V, Prof.A M Deshmukh, "Channel Estimation in OFDM", International Journal of Engineering Research and Applications (IJERA) ,Vol. 3, Issue 1, January -February 2013, pp.14131415 . 